\title{
Structural pathology is not related to patient- reported pain and function in patients undergoing meniscal surgery
}

\author{
Simon Maretti Tornbjerg, ${ }^{1,2}$ Nis Nissen, ${ }^{2}$ Martin Englund, ${ }^{3,4}$ Uffe Jørgensen, ${ }^{5}$ \\ Jeppe Schjerning, ${ }^{6}$ L Stefan Lohmander, $^{7}$ Jonas Bloch Thorlund ${ }^{1}$
}

\begin{abstract}
- Additional material is published online only. To view please visit the journal online (http://dx.doi.org/10.1136/ bjsports-2016-096456)
\end{abstract}

For numbered affiliations see end of article.

\section{Correspondence to} Jonas Bloch Thorlund, Department of Sports Science and Clinical Biomechanics University of Southern Denmark Campusvej 55, Odense M 5230, Denmark; jthorlund@health.sdu.dk

Accepted 30 August 2016 Published Online First 16 September 2016

\section{CrossMark}

$$
\begin{aligned}
& \text { To cite: Tornbjerg SM, } \\
& \text { Nissen N, Englund M, } \\
& \text { et al. Br J Sports Med } \\
& \text { 2017:51:525-530. }
\end{aligned}
$$

\begin{abstract}
Background The relationship between meniscal tears and other joint pathologies with patient-reported symptoms is not clear. We investigated associations between structural knee pathologies identified at surgery with preoperative knee pain and function in patients undergoing arthroscopic meniscal surgery.

Methods This study included 443 patients from the Knee Arthroscopy Cohort Southern Denmark (KACS), a prospective cohort following patients 18 years or older undergoing arthroscopic meniscal surgery at 4 hospitals between 1 February 2013 and 31 January 2014.

Patient-reported outcomes, including the Knee Injury and Osteoarthritis Outcome Score (KOOS), were obtained by online questionnaires prior to surgery. Knee pathology was assessed by the operating surgeons using a modified version of the International Society of Arthroscopy, Knee Surgery and Orthopaedic Sports Medicine (ISAKOS) classification of meniscal tears questionnaire, supplemented with information extracted from surgery reports. Following hypothesis-driven preselection of candidate variables, backward elimination regressions were performed to investigate associations between patient-reported outcomes and structural knee pathologies.
\end{abstract}

Results Regression models only explained a small proportion of the variability in self-reported pain and function (adjusted $R^{2}=0.10-0.12$ ) and this association was mainly driven by age, gender and body mass index. Conclusions Specific meniscal pathology and other structural joint pathologies found at meniscal surgery were not associated with preoperative self-reported pain and function in patients with meniscal tears questioning inferences made about a direct relationship between these. Our findings question the role of arthroscopic surgery to address structural pathology as a means to improve patient-reported outcomes in patients having surgery for a meniscal tear.

\section{INTRODUCTION}

Arthroscopic meniscal surgery is one of the most commonly performed orthopaedic procedures. ${ }^{1}$ Diagnosis of meniscal tears involves clinical history and physical examination and is often confirmed by MRI, which has high accuracy in diagnosing meniscal tears. ${ }^{2-4}$ MRI-based studies have reported that meniscal tears are common in the contralateral asymptomatic knee in patients sustaining a knee trauma ${ }^{5}$ and in middle-aged and older individuals without symptoms and/or signs of radiographic knee osteoarthritis. ${ }^{6-8}$ In combination with recent evidence challenging the effect of knee arthroscopy for meniscal tears, ${ }^{9}$ the theoretical framework for meniscal surgery has been questioned: that the meniscus tear is responsible for the patient perceived pain and other symptoms, and that dealing with the meniscal tear by surgery will lead to resolution of symptoms. ${ }^{10}$

Meniscal tears often present in combination with other knee pathology at arthroscopy. Thus, it is plausible that knee symptoms may be caused by factors other than meniscal tears per se, ${ }^{11}$ or some types of meniscal tears could be symptomatic while others are not. Arthroscopy can provide information about meniscal tear pattern and coexisting pathologies potentially related to knee symptoms, such as cartilage damage, synovial changes and inflammation. ${ }^{12}$

The aim of this study was to investigate the association between structural knee pathologies identified at surgery with preoperative pain and function in patients with a verified meniscal tear at surgery.

\section{MATERIALS AND METHODS}

\section{Study design}

Cross-sectional study investigating the association between preoperative patient-reported knee pain and function and pathological findings at knee arthroscopy.

\section{Participants}

Participants from the Knee Arthroscopy Cohort Southern Denmark (KACS) ${ }^{13}$ with baseline assessment of patient-reported outcomes and a verified meniscal tear at surgery. KACS is a prospective cohort study following patients recruited between 1 February 2013 and 31 January 2014 undergoing knee arthroscopy for a meniscal tear at four different public hospitals in Denmark. Patients were informed about the KACS cohort study by a study nurse and asked to sign the consent form after being referred to surgery for a meniscal tear by an orthopaedic surgeon. For logistical reasons, participants at one study site were presented with information about the study prior to seeing the orthopaedic surgeon but signed the consent form after being referred to surgery.

Inclusion criteria were: $\geq 18$ years of age, referred to knee arthroscopy on suspicion of a meniscus tear based on clinical examination by an orthopaedic surgeon and MRI (if considered necessary), able to read and speak Danish and having an email address.

Exclusion criteria were: no meniscal tear found at surgery, previous or planned ACL or posterior 
cruciate ligament reconstruction surgery in either knee, fracture (s) to the lower extremities within the past 6 months prior to recruitment or not able to reply to the questionnaire because of mental impairment. Patients with missing data on any structural pathology were excluded from this study.

Informed consent to the use of personal data was obtained from all included patients, even though the Regional Scientific Ethics Committee of Southern Denmark waived the need for ethical approval after reviewing the outline of KACS. ${ }^{13}$

\section{Patient-reported outcomes}

Patient-reported outcomes were collected using online questionnaires prior to surgery (median 7 days, IQR 3-11 days). The patient-reported outcomes (ie, dependent variables) of interest for this analysis were: pain, function in sport and recreation (Sport/Rec) and function in daily living (ADL) assessed using the Knee Injury and Osteoarthritis Outcome Score (KOOS). ${ }^{14}$ These domains were selected, based on their inclusion as primary outcomes to study the effectiveness of meniscal surgery in previous studies. ${ }^{15}$ KOOS is a knee-specific questionnaire used to assess patient-reported outcomes of knee injury consisting of five subscales in total: pain, symptoms, ADL, Sport/Rec and quality of life. Each domain ranges from 0 to 100 points with 0 being extremely symptomatic and 100 being asymptomatic. The inclusion of subscales of physical function relating to daily life and sport and recreation enhances KOOS's validity for patients within a wide age range and range of current and expected physical activity levels, ${ }^{16}$ such as patients undergoing meniscus surgery since these range from young to old. KOOS is valid for patients undergoing arthroscopic meniscal surgery, ${ }^{14}{ }^{16-18}$ and has been used to assess self-reported outcomes in this group of patients. $^{19} 20$

\section{Structural pathology at arthroscopy}

Objective findings of knee pathologies pertaining to the meniscus and cartilage were recorded by the operating surgeon at arthroscopy using a modified version of the International Society of Arthroscopy, Knee Surgery and Orthopaedic Sports Medicine (ISAKOS) classification of meniscal tears questionnaire $^{21}$ (ie, including scoring of cartilage using the International Cartilage Repair Society (ICRS) grading system). ${ }^{22}$ This information was supplemented with additional pathological findings extracted from the patients' surgery reports. Information registered by surgeons on the modified ISAKOS questionnaire was transferred from paper format to electronic format using automated forms processing. This method is a valid alternative to double entry of data. ${ }^{23}$

The following variables were included in the analysis: tear location (ie, medial and/or lateral tear), tear depth, circumferential location of tear, radial location of tear, tear pattern, meniscus tissue quality, length of tear, cartilage grade, presence of plica and knee joint laxity assessed by the surgeon at surgery. ACL rupture (only on non-reconstructed patients) and grade of inflammation/synovitis were retrieved from surgery reports. Synovitis was generally well described, especially when present, therefore missing descriptions of synovitis were considered as no synovitis present.

\section{Variable grouping}

To improve interpretability of statistical models, we grouped the selected independent variables into blocks of variables of similar construct. For example, all variables concerning structural change of the meniscus were grouped together in a single block. Thus, all selected variables were entered into one of five blocks: previous surgery, meniscal pathology, cartilage pathology, other findings at arthroscopy and patients characteristics (ie, age, sex and body mass index (BMI) as they are considered traditional confounders; see online supplementary appendix table S1).

\section{Statistical analysis}

No power calculation was conducted due to the exploratory nature of the study. Before exclusion of patients with missing data, we conducted a drop out analysis to test for differences between patients with and without missing data. Continuous data were tested using student's unpaired t-test and categorical data using either the $\chi^{2}$ or Fischer's exact test.

Prior to analysis, collinearity was investigated by calculating variance inflation factors (VIFs) for all independent variables. The level of collinearity was not considered problematic if mean VIF was below 4 and individual VIFs were $<10 .{ }^{24}$ For each outcome (ie, pain, Sport/Rec and ADL), we performed a linear backward elimination regression analysis on prespecified blocks of variables to investigate which pathological factors that were related to the three different outcomes, respectively. Additionally, we assessed the magnitude and direction of associations between outcome measures and variables included in the models. At first, all candidate blocks were included in the model. At each step, the least significant block of variables was removed by backward elimination using a cut-off of $\mathrm{p} \leq 0.10$.

$\mathrm{R}^{2}$ and adjusted $\mathrm{R}^{2}$ were examined to describe how well the models explained the overall variance. In addition, hierarchical regressions were performed for blocks reaching statistical significance (ie, $\mathrm{p} \leq 0.10$ ). Significant blocks were entered in order from lowest to highest $\mathrm{p}$ value to assess individual $\mathrm{R}^{2}$ for each block. This method was also used to assess $\mathrm{R}^{2}$ values for individual variables within each significant block. Finally, distribution of residuals and level of heteroskedasticity were checked for each backward elimination regression model to determine how well a linear regression described the data. Distribution of

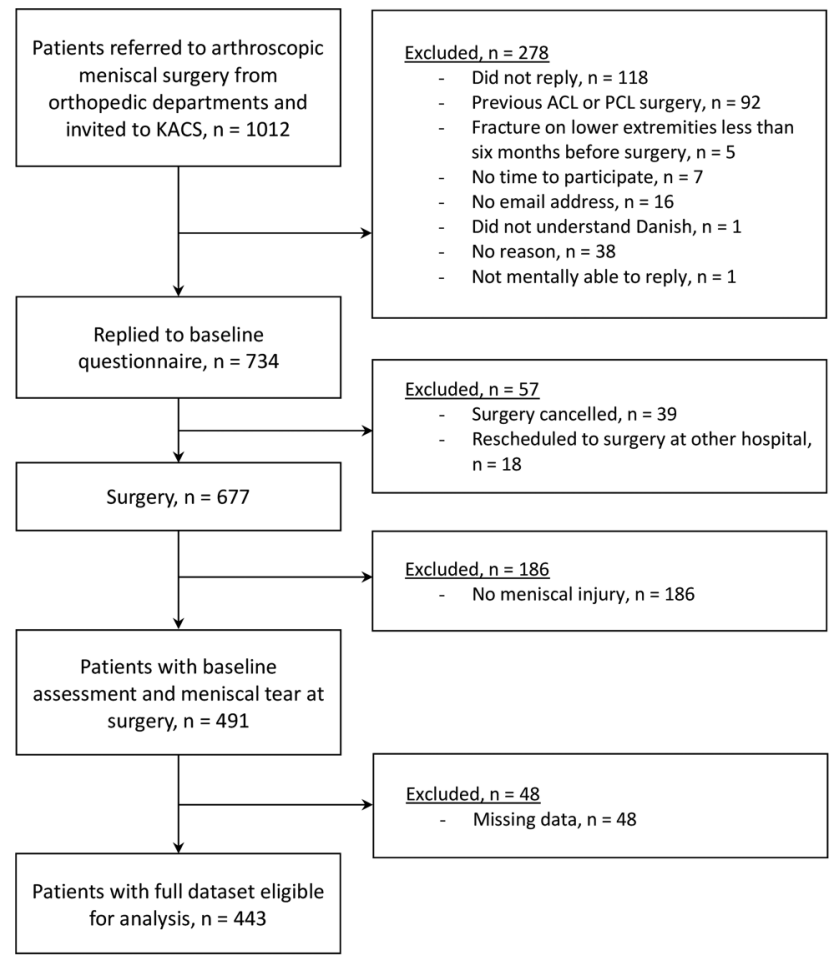

Figure 1 Flow chart of inclusion. 
Table 1 Characteristics of patients

\begin{tabular}{|c|c|c|}
\hline \multirow[b]{2}{*}{ Variables } & \multicolumn{2}{|l|}{$\mathrm{N}=443$} \\
\hline & & Range \\
\hline Age, years (SD) & $49.7(12.8)$ & $18-76$ \\
\hline Sex, female, n (\%) & $195(44.0)$ & \\
\hline Height, cm (SD) & $174.9(9.5)$ & 152-201 \\
\hline Weight, kg (SD) & $83.7(15.6)$ & $48-149$ \\
\hline$B M I, \mathrm{~kg} / \mathrm{m}^{2}(\mathrm{SD})$ & $27.3(4.3)$ & $19.5-47.2$ \\
\hline \multicolumn{3}{|l|}{ KOOS, (SD) } \\
\hline Pain & $54.5(18.7)$ & $0-97$ \\
\hline Sport and recreation & $25.8(21.5)$ & $0-100$ \\
\hline$A D L$ & $63.6(19.5)$ & $3-100$ \\
\hline Previous meniscus surgery on index knee, $\mathrm{n}(\%)$ & $79(17.8)$ & \\
\hline \multicolumn{3}{|l|}{ Tear location } \\
\hline Medial & $329(74.3)$ & \\
\hline Lateral & $74(16.7)$ & \\
\hline Both & $40(9.0)$ & \\
\hline \multicolumn{3}{|l|}{ Tear pattern, n (\%) } \\
\hline Longitudinal-vertical (bucket handle) & $84(19.0)$ & \\
\hline Horizontal & $32(7.2)$ & \\
\hline Radial & $29(6.6)$ & \\
\hline Vertical flap & $105(23.7)$ & \\
\hline Horizontal flap & $24(5.4)$ & \\
\hline Complex & $118(26.6)$ & \\
\hline Root tear & $2(0.5)$ & \\
\hline More than one tear type & $49(11.1)$ & \\
\hline \multicolumn{3}{|l|}{ Tissue quality, n (\%) } \\
\hline Non-degenerative & $168(37.9)$ & \\
\hline Degenerative & $261(58.9)$ & \\
\hline Undetermined & $14(3.2)$ & \\
\hline Tear length, mm (SD) & $16.9(8.7)$ & $2-50$ \\
\hline \multicolumn{3}{|l|}{ Tear depth, n (\%) } \\
\hline Partial & $164(37.0)$ & \\
\hline Complete & $279(63.0)$ & \\
\hline \multicolumn{3}{|l|}{ Radial location, $\mathrm{n}(\%)$} \\
\hline Posterior & $288(65.0)$ & \\
\hline Mid body & $44(9.9)$ & \\
\hline Posterior+mid body & $73(16.5)$ & \\
\hline Anterior & $15(3.4)$ & \\
\hline Anterior+mid body & $4(0.9)$ & \\
\hline Posterior+anterior & $1(0.2)$ & \\
\hline All & $18(4.1)$ & \\
\hline \multicolumn{3}{|l|}{ Circumferential location, $\mathrm{n}(\%)$} \\
\hline Zone 1 & $139(31.4)$ & \\
\hline Zone 2 & $230(51.9)$ & \\
\hline Zone 3 & $74(16.7)$ & \\
\hline \multicolumn{3}{|l|}{ Medial cartilage grade, $\mathrm{n}(\%)$} \\
\hline Grade 0 & $111(25.1)$ & \\
\hline Grade 1 & $107(24.2)$ & \\
\hline Grade 2 & $89(20.1)$ & \\
\hline Grade 3 & $101(22.8)$ & \\
\hline Grade 4 & $35(7.9)$ & \\
\hline \multicolumn{3}{|l|}{ Lateral cartilage grade, $\mathrm{n}(\%)$} \\
\hline Grade 0 & $166(37.5)$ & \\
\hline Grade 1 & $153(34.5)$ & \\
\hline Grade 2 & 78 (17.6) & \\
\hline Grade 3 & $35(7.9)$ & \\
\hline Grade 4 & $11(2.5)$ & \\
\hline \multicolumn{3}{|l|}{ Patellofemoral cartilage grade, $\mathrm{n}(\%)$} \\
\hline Grade 0 & $155(35.0)$ & \\
\hline
\end{tabular}

Table 1 Continued

\begin{tabular}{lc}
\hline & $\mathrm{N}=443$ \\
\cline { 2 - 2 } Variables & Range \\
\hline Grade 1 & $114(25.7)$ \\
Grade 2 & $77(17.4)$ \\
Grade 3 & $70(15.8)$ \\
Grade 4 & $27(6.1)$ \\
Plica, $\mathrm{n}(\%)$ & $165(37.3)$ \\
Synovitis, $\mathrm{n}$ (\%) & \\
None & $247(55.8)$ \\
Irritation/redness & $37(8.4)$ \\
Light synovitis & $100(22.6)$ \\
Moderate synovitis & $41(9.3)$ \\
Severe synovitis & $18(4.1)$ \\
ACL, $\mathrm{n}$ (\%) & \\
Intact & $394(89.0)$ \\
Partial rupture (non-reconstructed) & $17(3.8)$ \\
Total rupture (non-reconstructed) & $32(7.2)$ \\
Knee joint laxity (surgeon assessed), n (\%) & \\
No laxity & $384(86.7)$ \\
Slight laxity & $44(9.9)$ \\
Pronounced laxity & $15(3.4)$ \\
\hline Continuous variables are reported as means & \\
frequencies and percentages. & \\
Percentages may not add up to 100\% due to rounding. & \\
ADL, function in daily living; BMI, body mass index; KOOS, Knee Injury and \\
Osteoarthritis Outcome Score. & \\
\hline
\end{tabular}

residuals was inspected visually using histograms and qq-plots. Level of heteroskedasticity was inspected visually by plotting standardised residuals against fitted values.

\section{RESULTS}

\section{Participant characteristics}

In total, 1012 patients were invited to participate in the study. Three hundred and thirty-five of these were excluded prior to surgery, and 186 out of the 677 patients who underwent arthroscopic surgery were excluded because no meniscal tear was found (ie, corresponding to $18 \%, 12 \%, 30 \%$ and $16 \%$ of patients at the four recruitment sites, respectively; figure 1). The majority $(71 \%)$ of the remaining 491 patients had a preoperative MRI to confirm the presence of a meniscal tear. The 491 arthroscopic surgeries were performed by 42 different surgeons, with 15 surgeons being responsible for $86 \%$ of the surgeries. Subsequent to surgery, 48 patients with missing values on the modified ISAKOS questionnaire were excluded after no difference was found between patients with and without missing data (see online supplementary appendix table S2). After exclusions, a total of 443 patients were included for analysis (figure 1).

The majority of patients were male and the study sample on average middle-aged and slightly overweight. Most patients had medial meniscal tears and many patients had concomitant cartilage damage (table 1).

\section{Regression analyses}

In general, no association between structural pathology and selfreported outcomes was found for pain or ADL function (tables 2 and 3). For Sport/Rec function, $<5 \%$ of the variability in this score could be attributed to the 'other findings at arthroscopy', which was driven by increasing levels of synovitis being 
Table 2 Backward stepwise regression in blocks for associations with KOOS pain

\begin{tabular}{|c|c|c|c|c|c|}
\hline Variables & $\begin{array}{l}\text { Adjusted } \\
\mathbf{R}^{2}\end{array}$ & $\mathbf{R}^{2}$ & $\begin{array}{l}\text { Regression } \\
\text { coefficient } \\
(\beta)\end{array}$ & $95 \% \mathrm{Cl}$ & p Value \\
\hline KOOS pain & 0.100 & 0.107 & & & \\
\hline $\begin{array}{l}\text { Person } \\
\text { characteristics }\end{array}$ & & 0.107 & & & $<0.001$ \\
\hline Age & & 0.014 & -0.18 & $(-0.31$ to -0.05$)$ & 0.008 \\
\hline Sex, female & & 0.037 & -6.69 & $(-10.06$ to -3.33$)$ & $<0.001$ \\
\hline BMI & & 0.055 & -0.96 & $(-1.34$ to -0.57$)$ & $<0.001$ \\
\hline $\begin{array}{l}\text { Model } \\
\text { intercept }\end{array}$ & & & 92.4 & (80.4 to 104.3 ) & \\
\hline
\end{tabular}

Elimination criteria $\mathrm{p} \leq 0.10$

Stepwise elimination of blocks of independent variables: Previous meniscus surgery $(p=0.908)$, other findings at arthroscopy $(p=0.441)$, meniscal pathology $(p=0.308)$, cartilage pathology ( $p=0.372$ ).

Individual $R^{2}$ values may not add up to total $R^{2}$ due to rounding

BMI, body mass index; KOOS, Knee Injury and Osteoarthritis Outcome Score.

Table 3 Backward stepwise regression in blocks for associations with KOOS ADL

\begin{tabular}{|c|c|c|c|c|c|}
\hline Variables & $\begin{array}{l}\text { Adjusted } \\
\mathbf{R}^{2}\end{array}$ & $\mathbf{R}^{2}$ & coefficient $(\boldsymbol{\beta})$ & $95 \% \mathrm{Cl}$ & p Value \\
\hline KOOS ADL & 0.116 & 0.122 & & & \\
\hline $\begin{array}{l}\text { Person } \\
\text { characteristics }\end{array}$ & & 0.122 & & & $<0.001$ \\
\hline Age & & 0.044 & -0.30 & $(-0.43$ to -0.16$)$ & $<0.001$ \\
\hline Sex, female & & 0.021 & -5.77 & $(-9.24$ to -2.30$)$ & 0.001 \\
\hline BMI & & 0.056 & -0.97 & $(-1.37$ to -0.58$)$ & $<0.001$ \\
\hline $\begin{array}{l}\text { Model } \\
\text { intercept }\end{array}$ & & & 107.4 & (95.0 to 119.7 ) & \\
\hline \multicolumn{6}{|c|}{$\begin{array}{l}\text { Elimination criteria } p \leq 0.10 \text {. } \\
\text { Stepwise elimination of blocks of independent variables: previous meniscus } \\
(p=0.926) \text {, other findings at arthroscopy }(p=0.353) \text {, meniscal pathology }(p= \\
\text { cartilage pathology ( } p=0.308) \text {. } \\
\text { Individual } R^{2} \text { values may not add up to total } R^{2} \text { due to rounding. } \\
\text { ADL, function in daily living; BMI, body mass index; KOOS, Knee Injury and } \\
\text { Osteoarthritis Outcome Score. }\end{array}$} \\
\hline
\end{tabular}

associated with poorer Sport/Rec function (table 4). The 'other findings at arthroscopy' block was the only significant structural pathology block across models.

Overall, the adjusted $\mathrm{R}^{2}$ values indicated that the variables in the regression models could explain only 10\%, 11\% and $12 \%$ of the variability in Pain, Sport/Rec and ADL, respectively, and this was mainly explained by the patient characteristics age, gender and BMI (tables 2-4).

\section{Collinearity and model assumptions}

No collinearity was detected between independent variables included in the regression analyses. Visual inspection of relevant plots and appropriate statistical tests showed normal distribution of residuals and no signs of heteroskedasticity for the pain and ADL model, confirming that a linear regression described the models well. The Sport/Rec model showed obvious flooring effect, slight heteroskedasticity and a less perfect distribution of residuals than the other models, however the linear regression model still described the data sufficiently.

\section{DISCUSSION}

We found no relevant association between structural knee pathology and self-reported pain and function prior to arthroscopic meniscal surgery in this study. Synovitis was the only variable to explain a very small part of the variability in the self-reported Sport/Rec function of the KOOS questionnaire. In all models, the patient characteristics of age, female gender and greater BMI were consistently but weekly associated with poorer self-reported outcomes.

MRI-based studies have reported that the presence of meniscal tears is common in patient knees in the absence of patientreported symptoms, ${ }^{5-8}$ challenging the classical tenet of meniscal injuries being a main cause of knee symptoms in patients with meniscal pathology. The menisci are mainly innervated in the peripheral, vascular zone, represented by the outer one-third of the menisci and the anterior and posterior horns. ${ }^{25}$ No nerve fibres are seen in the avascular intermediate or inner third of the menisci. ${ }^{25}$ Sensation of pain in the non-anaesthetised human knee has been reported to be markedly higher from the outer than the inner rim of the menisci, investigated by instrumented arthroscopic palpation. ${ }^{26}$ Thus, it is plausible that factors such as size, location and type of meniscal tear could be important for patient perceived pain. Hypothetically, a small radial tear in the avascular zone should not cause pain, whereas a tear in the outer third part presumably would. However, in the present study we found no association between any of the investigated structural pathologies related to the meniscal tear and selfreported pain.

Synovitis has been reported to be associated with preoperative pain and dysfunction in patients with traumatic meniscal injury undergoing partial meniscectomy. ${ }^{12}$ The results of the present study are in line with this, as increasing levels of synovitis were somewhat associated with poorer function during strenuous tasks (ie, Sport/Rec function). However, this result should be interpreted with caution as the association was not consistent across models and only explained about $3 \%$ of the variability in KOOS Sport/Rec function score.

\section{Implications of findings}

Inferences about a clear relationship between structural knee pathology and patient perceived symptoms as part of a rationale for the indication for arthroscopic meniscus surgery are not supported by results of the present study. Further, the results of our study do not support a construct of specific subgroups of patients, based on meniscal and other structural pathology, which may have particular benefit of surgery compared with others. The lack of association between structural pathology on the one hand, and pain and physical function on the other hand, may partially explain the reported lack of efficacy of arthroscopic surgery for knee pain in middle-aged and older adults. ${ }^{9}$

\section{Limitations}

The analysis was based on stepwise regression procedures, which are data driven and known to produce selection bias and optimism as a result of overfitting. ${ }^{27} 28$ However, models with small sample sizes are most prone to overfitting, ${ }^{28}$ and selected variables with very small $p$ values (for instance $p<0.001$ ) are much less prone to selection bias and overfitting than variables with $\mathrm{p}$ values near the chosen significance level. ${ }^{27}$ Yet, no internal validation procedure was performed in this study, and the extent of overfitting due to the use of predictor selection strategies is therefore unaccounted for. ${ }^{28}$ Given the general lack 
Table 4 Backward stepwise regression in blocks for associations with KOOS Sport/Rec

\begin{tabular}{|c|c|c|c|c|c|}
\hline Variables & Adjusted $\mathbf{R}^{2}$ & $\mathbf{R}^{2}$ & $\begin{array}{l}\text { Regression } \\
\text { coefficient }(\boldsymbol{\beta})\end{array}$ & $95 \% \mathrm{Cl}$ & p Value \\
\hline KOOS Sport/Rec & 0.113 & 0.137 & & & \\
\hline Person characteristics & & 0.094 & & & $<0.001$ \\
\hline Age & & 0.027 & -0.23 & $(-0.39$ to -0.07$)$ & 0.005 \\
\hline Sex, female & & 0.058 & -8.98 & $(-12.89$ to -5.07$)$ & $<0.001$ \\
\hline BMI & & 0.009 & -0.40 & $(-0.84$ to 0.05$)$ & 0.081 \\
\hline Other findings at arthroscopy & & 0.043 & & & 0.013 \\
\hline Synovitis & & 0.030 & & & 0.011 \\
\hline None & & & Reference & & \\
\hline Irritation/redness & & & -3.50 & $(-10.63$ to 3.63$)$ & \\
\hline Mild synovitis & & & -6.05 & $(-10.85$ to -1.25$)$ & \\
\hline Moderate synovitis & & & -9.00 & $(-15.99$ to -2.01$)$ & \\
\hline Severe synovitis & & & -11.21 & $(-21.04$ to -1.39$)$ & \\
\hline $\mathrm{ACL}$ rupture & & 0.001 & & & 0.800 \\
\hline Intact & & & Reference & & \\
\hline Partial rupture (non-reconstructed) & & & -3.43 & $(-14.29$ to 7.43$)$ & \\
\hline Total rupture (non-reconstructed) & & & 0.38 & $(-9.48$ to 10.24$)$ & \\
\hline Plica & & 0.001 & & & 0.493 \\
\hline No & & & Reference & & \\
\hline Yes & & & 1.40 & $(-2.61$ to 5.42$)$ & \\
\hline Knee joint laxity (surgeon assessed) & & 0.010 & & & 0.259 \\
\hline No laxity & & & Reference & & \\
\hline Slight laxity & & & -5.61 & $(-12.98$ to 1.76$)$ & \\
\hline Pronounced laxity & & & -7.48 & $(-20.64$ to 5.68$)$ & \\
\hline Model intercept & & & 54.9 & (40.8 to 69.0$)$ & \\
\hline
\end{tabular}

of relationship between structural pathology and self-reported outcomes in this study, we do not consider overfitting of models a problem. To accommodate for possible variable selection bias, we narrowed the list of candidate variables prior to backward elimination regressions based on literature and likely hypotheses, making the models hypothesis driven. Such preanalysis selection is considered a good alternative to plain data-driven variable selection. ${ }^{28}$ To avoid biased selection of variables prior to analysis, no statistical tests were conducted on the data in advance of the selection procedure.

In the present study, we used validated methods ${ }^{21} 22$ to systematically collect information about knee joint pathology at arthroscopy supplemented with information from surgery reports. Even though such methods have shown moderate-to-good reliability, some misclassification of joint pathology may have occurred, which potentially could attenuate an association with self-reported pain and function. However, we consider it unlikely that such misclassification may have caused the complete lack of association observed in the present study.

In the present study, 186 of 677 patients who underwent surgery on suspicion of a meniscal tear did not have a meniscal tear at arthroscopy leading them to being excluded from our present analysis. This may partly be explained by the absence of MRI to verify the presence of a meniscal tear in all patients. However, we believe the results are generalisable to Danish patients undergoing meniscal surgery as the average age of patients in the present study was 49.7 years and $44 \%$ were female. This is similar to nationwide data from the Danish National Patient Registry showing that in 2011 patients undergoing meniscal surgery on average were 47 years old and $41 \%$ were female. ${ }^{29}$

\section{CONCLUSION}

Specific meniscal pathology and other structural joint pathologies found at arthroscopic meniscal surgery were not associated with preoperative self-reported pain and function in patients with meniscal tears, suggesting that inferences about a direct relationship between these are questionable. Increasing age, female gender and greater BMI were consistently associated with poorer self-reported outcomes, although the variability explained was modest. Our findings question the role of arthroscopic surgery to address structural pathology as a means to improve patient-reported outcomes in patients having surgery for a meniscal tear.

\section{What are the findings?}

- Meniscal pathology features such as tear type, tear size and tear location identified at arthroscopy were not associated with preoperative self-reported knee pain and function in patients undergoing arthroscopic meniscal surgery.

- Other structural knee joint pathologies found at surgery, such as cartilage damage, were not associated with preoperative self-reported knee pain and function.

- Increasing age, female gender and greater body mass index were modestly associated with poorer self-reported outcomes. 


\section{How might it impact on clinical practice in the future?}

- These findings question inferences made about relationships between structural knee pathology and patient perceived symptoms.

- Our findings question the role of arthroscopy to address structural pathology as a means to improve patient-reported outcomes and the construct of specific subgroups of patients, based on meniscal and other structural pathology, who may benefit from meniscal surgery.

\author{
Author affiliations \\ ${ }^{1}$ Department of Sports Science and Clinical Biomechanics, University of Southern \\ Denmark, Odense, Denmark \\ ${ }^{2}$ Department of Orthopaedics, Lillebaelt Hospital, Kolding, Denmark \\ ${ }^{3}$ Faculty of Medicine, Department of Clinical Sciences Lund, Orthopaedics, Clinical \\ Epidemiology Unit, Lund University, Lund, Sweden \\ ${ }^{4}$ Clinical Epidemiology Research and Training Unit, Boston University School of \\ Medicine, Boston, Massachusetts, USA \\ ${ }^{5}$ Department of Orthopaedics and Traumatology, Odense University Hospital, \\ Odense, Denmark \\ ${ }^{6}$ Department of Orthopaedics, Lillebaelt Hospital, Vejle, Denmark \\ ${ }^{7}$ Faculty of Medicine, Department of Clinical Sciences Lund, Orthopaedics, \\ Lund University, Lund, Sweden
}

Acknowledgements The authors would like to acknowledge the efforts of all participating patients and orthopaedic surgeons, nurses and secretaries at the Department of Orthopedics and Traumatology, Odense University Hospital (Odense and Svendborg) and the Department of Orthopedics, Lillebaelt Hospital (Kolding and Vejle) for their assistance with patient recruitment and data collection. Also they would like to thank statistician Aleksandra Turkiewicz, Lund University, Faculty of Medicine, Department of Clinical Sciences Lund, Orthopaedics, Clinical Epidemiology Unit, Lund, Sweden for input on the statistical models.

Contributors SMT, JBT, SL and ME conceived and designed the study. NN, UJ and JS participated in the setup of the study, patient recruitment and data collection. SMT and JBT conducted the analysis and/or interpretation. SMT and JBT drafted the first version of the manuscript. All authors helped in revising the manuscript and gave their final approval of the submitted version.

Funding This study was supported by an individual postdoctoral grant (JBT) from the Danish Council for Independent Research | Medical Sciences, funds from The Region of Southern Denmark, funds from the Department of Orthopaedics, Lillebaelt Hospital, Kolding and a grant from Lillebaelt Hospital Research Fund.

Competing interests None declared.

Ethics approval The Regional Scientific Ethics Committee of Southern Denmark. Provenance and peer review Not commissioned; externally peer reviewed.

\section{REFERENCES}

1 Cullen KA, Hall MJ, Golosinskiy A. Ambulatory surgery in the United States, 2006. Natl Health Stat Report 2009(11):1-25.

2 Crawford R, Walley G, Bridgman S, et al. Magnetic resonance imaging versus arthroscopy in the diagnosis of knee pathology, concentrating on meniscal lesions and ACL tears: a systematic review. Br Med Bull 2007;84:5-23.

3 Subhas N, Sakamoto FA, Mariscalco MW, et al. Accuracy of MRI in the diagnosis of meniscal tears in older patients. AJR Am J Roentgenol 2012;198: W575-80.

4 Van Dyck P, Vanhoenacker FM, Lambrecht V, et al. Prospective comparison of 1.5 and 3.0-T MRI for evaluating the knee menisci and ACL. J Bone Joint Surg Am 2013;95:916-24.
5 Boks SS, Vroegindeweij D, Koes BW, et al. Magnetic resonance imaging abnormalities in symptomatic and contralateral knees: prevalence and associations with traumatic history in general practice. Am J Sports Med 2006;34:1984-91.

6 Englund M, Guermazi A, Gale D, et al. Incidental meniscal findings on knee MRI in middle-aged and elderly persons. N Eng/ J Med 2008;359:1108-15.

7 Bhattacharyya T, Gale D, Dewire P, et al. The clinical importance of meniscal tears demonstrated by magnetic resonance imaging in osteoarthritis of the knee. J Bone Joint Surg Am 2003;85-A:4-9.

8 Guermazi A, Niu J, Hayashi D, et al. Prevalence of abnormalities in knees detected by MRI in adults without knee osteoarthritis: population based observational study (Framingham Osteoarthritis Study). BMJ 2012;345:e5339.

9 Thorlund JB, Juhl CB, Roos EM, et al. Arthroscopic surgery for degenerative knee: systematic review and meta-analysis of benefits and harms. BMJ 2015;350:h2747.

10 Aspenberg P. Mythbusting in orthopedics challenges our desire for meaning. Acta Orthop 2014;85:547.

11 Englund M, Niu J, Guermazi A, et al. Effect of meniscal damage on the development of frequent knee pain, aching, or stiffness. Arthritis Rheum 2007; 56:4048-54.

12 Scanzello CR, McKeon B, Swaim BH, et al. Synovial inflammation in patients undergoing arthroscopic meniscectomy: molecular characterization and relationship to symptoms. Arthritis Rheum 2011;63:391-400.

13 Thorlund JB, Christensen R, Nissen N, et al. Knee Arthroscopy Cohort Southern Denmark (KACS): protocol for a prospective cohort study. BMJ Open 2013;3: e003399.

14 Roos EM, Roos HP, Lohmander LS, et al. Knee Injury and Osteoarthritis Outcome Score (KOOS) - development of a self-administered outcome measure. J Orthop Sports Phys Ther 1998;28:88-96.

15 Khan M, Evaniew N, Bedi A, et al. Arthroscopic surgery for degenerative tears of the meniscus: a systematic review and meta-analysis. CMAJ 2014;186:1057-64.

16 Roos EM, Roos HP, Lohmander LS. WOMAC Osteoarthritis Index-additional dimensions for use in subjects with post-traumatic osteoarthritis of the knee. Western Ontario and MacMaster Universities. Osteoarthritis Cartilage 1999; 7:216-21.

17 Roos EM, Roos HP, Ekdahl C, et al. Knee injury and Osteoarthritis Outcome Score (KOOS) - validation of a Swedish version. Scand I Med Sci Sports 1998;8:439-48.

18 Roos EM, Toksvig-Larsen S. Knee injury and Osteoarthritis Outcome Score (KOOS) validation and comparison to the WOMAC in total knee replacement. Health Qual Life Outcomes 2003;1:17.

19 Herrlin S, Hållander M, Wange P, et al. Arthroscopic or conservative treatment of degenerative medial meniscal tears: a prospective randomised trial. Knee Surg Sports Traumatol Arthrosc 2007;15:393-401.

20 Herrlin SV, Wange PO, Lapidus G, et al. Is arthroscopic surgery beneficial in treating non-traumatic, degenerative medial meniscal tears? A five year follow-up. Knee Surg Sports Traumatol Arthrosc 2013;21:358-64.

21 Anderson AF, Irrgang JJ, Dunn W, et al. Interobserver reliability of the International Society of Arthroscopy, Knee Surgery and Orthopaedic Sports Medicine (ISAKOS) classification of meniscal tears. Am J Sports Med 2011;39:926-32.

22 Brittberg M, Winalski CS. Evaluation of cartilage injuries and repair. J Bone Joint Surg Am 2003;85-A(Suppl 2):58-69.

23 Paulsen A, Overgaard S, Lauritsen JM. Quality of data entry using single entry, double entry and automated forms processing - an example based on a study of patient-reported outcomes. PLOS ONE 2012;7:e35087.

24 Craney TA, Surles JG. Model-dependent variance inflation factor cutoff values. Qual Eng 2002;14:391-403.

25 Mine T, Kimura M, Sakka A, et al. Innervation of nociceptors in the menisci of the knee joint: an immunohistochemical study. Arch Orthop Trauma Surg 2000;120:201-4

26 Dye SF, Vaupel GL, Dye CC. Conscious neurosensory mapping of the internal structures of the human knee without intraarticular anesthesia. Am J Sports Med 1998:26:773-7.

27 Royston P, Moons KG, Altman DG, et al. Prognosis and prognostic research: developing a prognostic model. BMJ 2009;338:b604.

28 Moons KG, Altman DG, Reitsma JB, et al. Transparent reporting of a multivariable prediction model for Individual Prognosis or Diagnosis (TRIPOD): explanation and elaboration. Ann Intern Med 2015;162:W1-73.

29 Thorlund JB, Hare KB, Lohmander LS. Large increase in arthroscopic meniscus surgery in the middle-aged and older population in Denmark from 2000 to 2011. Acta Orthop 2014;85:287-92. 